\title{
Corrigendum
}

\section{Corrigendum to "Synergistic Effect of MiR-146a Mimic and Cetuximab on Hepatocellular Carcinoma Cells"}

\author{
Suning Huang $\mathbb{D},{ }^{1}$ Rongquan $H e \mathbb{D}^{2},{ }^{2}$ Minhua Rong $\mathbb{D},,^{3}$ Yiwu Dang $\mathbb{D},{ }^{4}$ and Gang Chen $\mathbb{D}^{4}$ \\ ${ }^{1}$ Department of Radiotherapy, First Affiliated Hospital of Guangxi Medical University, 6 Shuangyong Road, Nanning, \\ Guangxi Zhuang Autonomous Region 530021, China \\ ${ }^{2}$ Department of Medical Oncology, First Affiliated Hospital of Guangxi Medical University, 6 Shuangyong Road, Nanning, \\ Guangxi Zhuang Autonomous Region 530021, China \\ ${ }^{3}$ Research Department, Affiliated Cancer Hospital, Guangxi Medical University, 71 Hedi Road, Nanning, \\ Guangxi Zhuang Autonomous Region 530021, China \\ ${ }^{4}$ Department of Pathology, First Affiliated Hospital of Guangxi Medical University, 6 Shuangyong Road, Nanning, \\ Guangxi Zhuang Autonomous Region 530021, China \\ Correspondence should be addressed to Gang Chen; chen_gang_triones@163.com
}

Received 30 November 2020; Accepted 30 November 2020; Published 21 December 2020

Copyright (C) 2020 Suning Huang et al. This is an open access article distributed under the Creative Commons Attribution License, which permits unrestricted use, distribution, and reproduction in any medium, provided the original work is properly cited.

In the article titled, "Synergistic Effect of MiR-146a Mimic and Cetuximab on Hepatocellular Carcinoma Cells" [1], the authors reported that they had misspelt the name of a cell line and supplied the incorrect figure for publication. The correct name of the cell line should be Hep3B, instead of HepB3. The correct Figure 8 is displayed below. The results and conclusions described therein are not affected by these corrections. 


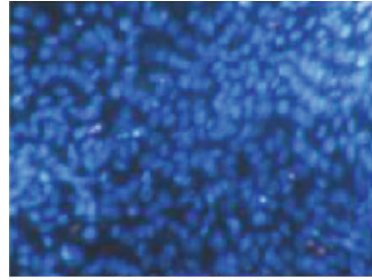

Mock control

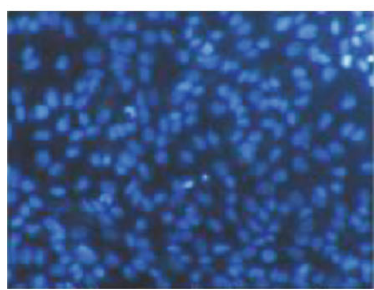

Negative mimic control

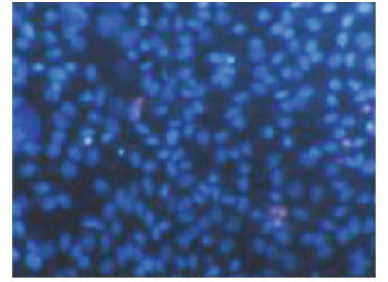

Negative inhibitor control

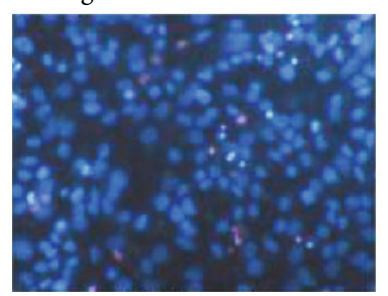

miR-146a mimic

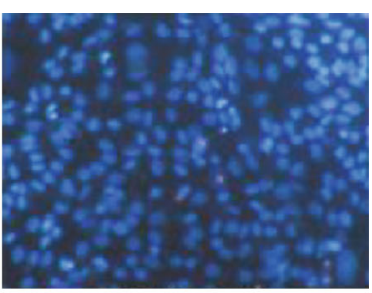

miR-146a inhibitor

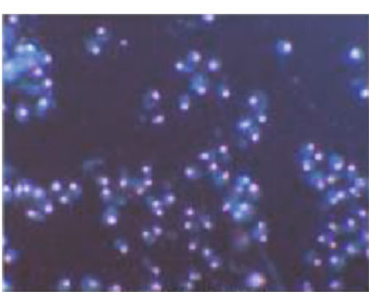

EGFR siRNA

FIGURE 8: MiR-146a mimic induced apoptosis with Hoechst 33342/propidium iodide (PI) double fluorescent chromatin staining. HepG2 cells $\left(2.5 \times 10^{3}\right.$ cells per well in a 96-well plate) were cultured for $24 \mathrm{~h}$ then transfected with miR-146a inhibitor, miR-146a mimic, EGFR siRNA, and their negative controls $(200 \mathrm{nM})$ up to another $96 \mathrm{~h}$. The effect on apoptosis was assessed and compared to mock and negative controls, $\times 200$.

\section{References}

[1] S. Huang, R. He, M. Rong, Y. Dang, and G. Chen, "Synergistic effect of miR-146a mimic and cetuximab on hepatocellular carcinoma cells," BioMed Research International, vol. 2014, Article ID 384121, 15 pages, 2014. 